\title{
LA FORMACIÓN DE LOS FUTUROS PROFESIONALES DE LOS GRADOS DE EDUCACIÓN, DE INFANTIL Y PRIMARIA, MEDIANTE LA FASE DE FORMACIÓN EN PRÁCTICAS Y SUS DESTREZAS DE ENSEÑANZA EFECTIVA
}

Pech Campos Silvia Joaquina

Prof. AD en la UCLM. Depto. de Psicología

Dra. en Filosofía y C. de la Educación. UCM silvia.pech@uclm.es

Ana Isabel Callejas Albiñana

Prof. AD en la UCLM. Depto. de Psicología

Dra. en Psicología, UCLM

Lilia González Velázquez

: Profesora Titular. UNACH. Dra. en Filosofía

y C. de la Educación, UCM

Fecha de Recepción: 8 Febrero 2019

Fecha de Admisión: 30 Abril 2019

\section{RESUMEN}

En este estudio se relaciona la significatividad de las prácticas supervisadas de formación de los Grados de Educación Infantil y Primaria, en las cohortes 2016-2017 y 2017-2018, con la autovaloración de la generación de las Destrezas - percibidas de Enseñanza Efectiva (EE). Se emplean mecanismos de Evaluación, de 360 grados, desde los aspectos del; Contexto, Entrada, Proceso y Producto, Modelo CIPP. Se realizan acciones con el fin de determinar y juzgar la eficacia de dicho proceso de formación en el perfil de competencias del profesional docente en los niveles mencionados.

Palabras claves: enseñanza afectiva; prácticum; grado de maestro en educación infantil; grado de maestro en educación primaria

\footnotetext{
ABSTRACT

This study relates the significance of the supervised training practices of the Degrees of Preschool and Primary Education, in the cohorts 2016-2017 and 2017-2018, with the self-assessment of the generation of the Skills-perceived of Effective Teaching (EE). Evaluation mechanisms are used, of 360 degrees, from the aspects of; Context, Input, Process and Product, CIPP Model. Actions are carried out in order to determine and judge the effectiveness of said training process in the professors' competency profile at the aforementioned levels.
} 


\section{LA FORMACIÓN DE LOS FUTUROS PROFESIONALES DE LOS GRADOS DE EDUCACIÓN, DE INFANTIL Y PRIMARIA, MEDIANTE LA FASE DE FORMACIÓN EN PRÁCTICAS Y SUS DESTREZAS DE ENSEÑANZA EFECTIVA}

Keywords: affective teaching; practicum; teacher's degree in early childhood education; grade of teacher in primary education

\section{OBJETIVOS DE LA INVESTIGACIÓN}

La presente propuesta se justifica en la definición de "estilo de enseñanza eficaz", como un conjunto organizado e interactivo de competencias docentes que se vertebran, a su vez, en un grupo de variables: autoeficacia, planificación, resolución de conflictos, comunicación y habilidades sociales, estudiado por autores como Valdivieso Burón, J. A.; Carbonero Martín, M. A.; Martín Antón, L. J.; Reoyo, N., (2012).

Se describe y analiza la formación en prácticas supervisadas de los Grados de Maestro en Educación, Infantil y Primaria y las Destrezas -percibidas de Enseñanza Efectiva. Se analizan las prácticas formativas con relación a las cuestiones siguientes:

¿En qué consiste la "buena enseñanza"?;

¿Es un Programa Educativo (PD) específico, de Maestro en Educación una carrera adecuada para desarrollar las Destrezas de Enseñanza Efectiva -EE?;

¿Qué problemas ayudará a resolver el estudio de la psicología educativa?;

¿Cómo apoya la psicología educativa el desarrollo de esas destrezas?;

¿Qué es lo que saben los maestros expertos?;

¿Cuáles son las principales preocupaciones de los maestros principiantes?;

¿Qué funciones cumplen en este campo la teoría, la investigación y la práctica en la formación de los profesionales de la educación?

Autores como Shön, Shulman, Zeichner, entre otros, enfatizan la importancia del logro de un Estándar de competencias profesionales y la reflexión sobre la práctica, para el desempeño profesional en el ámbito educativo en todos sus niveles.

Shulman, L., (1987) al ocuparse de esta cuestión, ha identificado siete áreas de conocimiento profesional. Los maestros expertos conocen:

Las materias académicas que enseñan;

Estrategias generales de enseñanza que aplican a todos los temas (como los principios que usted encontrará en este libro sobre el manejo del salón de clases, la enseñanza efectiva y la evaluación);

Los materiales y programas apropiados para su materia y el nivel en que la imparten;

Métodos concretos de enseñanza: formas especiales de enseñar conceptos particulares a ciertos estudiantes, como la mejor manera de explicar los números negativos a los alumnos menos capaces;

Las características y los antecedentes culturales de sus discípulos;

Los ambientes en que aprenden los estudiantes: en parejas, grupos pequeños, equipos, salones, escuelas y la comunidad;

Las metas y los propósitos de la enseñanza.

Un estándar profesional debe cubrir todos los aspectos mencionados.

\section{Las preocupaciones de los maestros principiantes}

Muchos profesores experimentan también lo que se ha llamado el "choque de la realidad" al presentarse a su primer empleo y enfrentar la "dura y cruel realidad de la vida diaria en el salón de clases", afirma Veenman, (1984). En general, los maestros principiantes comparten muchas preocupaciones. En una revisión de los estudios realizados en todo el mundo se descubrió que los maestros novatos señalan como sus principales problemas mantener la disciplina del aula, motivar a los estudiantes, resolver las diferencias entre los alumnos, evaluar su trabajo y tratar con los padres. 
Por otra parte, Darling-Hammond, L. (2012), propone el desarrollo de un enfoque sistémico para evaluar la docencia y fomentar una enseñanza eficaz.

Los estudios de Enseñanza Eficaz en el ámbito internacional de (Murillo Torrecilla, 2003), (Román, 2010). (Garrido \& Torrecilla, 2016), (Martínez-Garrido, 2015) los resume y propone un Decálogo de Enseñanza Eficaz (Murillo-Torrecilla, Martínez-Garrido, \& Hernández-Castilla, 2011).

Una de las importantes conclusiones sobre formación docente eficaz (Consejo Unión Europea, mayo 2014) es la de: Promover el desarrollo de marcos de competencias profesionales docentes1 que definan las competencias y cualidades que necesitan en las distintas etapas de sus carreras como docentes 0 en diferentes situaciones de enseñanza. Dichos marcos deben ser lo suficientemente flexibles como para responder a las necesidades cambiantes y ser debatidos en cooperación con las partes interesadas; así como fomentar el establecimiento de marcos de competencia profesional para los formadores de docentes, que establezcan las competencias que necesitan, al tiempo que refuercen la colaboración y el intercambio de prácticas entre pares y las áreas en desarrollo, como la mentoría en centros de los nuevos docentes.

Un número cada vez mayor de estados, en USA, basan sus requisitos en estándares desarrollados por INTASC, Interstate New Teacher Assessment and Support Consortium (2019). Se analizan dichos estándares propuestos para la contratación y el ejercicio de los profesionales de la educación en el ámbito de la universidad estadounidense, así como el modelo PRAXIS (Santrock, Woolfolk) que permite verificar la adquisición y el logro de dichas Destrezas de Enseñanza Efectiva.

Estos estándares se basan en 10 principios de enseñanza efectiva que INTASC ha identificado como esenciales para el aprendizaje óptimo de los estudiantes. Muchos estados evalúan los conocimientos que tienen los nuevos maestros de esos principios por medio de pruebas de las series PRAXIS IITM, publicadas por el Educational Testing Service. Dentro de las series PRAXIS IITM existen tres pruebas de Principles of Learning and Teaching (PLT) (Principios de Aprendizaje y Enseñanza), una de jardín de niños a sexto de primaria, otra de quinto de primaria a tercero de secundaria, y otra de primero de secundaria a tercero de bachillerato. Cada prueba PLT evalúa los conocimientos que tienen los estudiantes acerca de la psicología educativa y sus aplicaciones en el aula. (Woolfolk, A., 2010).

\section{MUESTRA Y/O PARTICIPANTES}

Se analiza la supervisión del proceso de la fase de formación en prácticas mediante el trabajo de los tutores en los CEIP y los tutores universitarios, mediante el seguimiento de las cohortes de Ios Grados MEI y MEP 2016-2017, 2017-2018. El periodo de Prácticum I del currículo del Grado en Educación de la UCLM, tiene una duración de 450 horas (18 ETCS), el cual se realiza durante el tercer curso, mientras que el periodo de Prácticum II, tiene una duración de 600 hrs. (24 créditos ETCS) y se realiza durante el cuarto curso.

Durante el curso académico 2017/2018 se matricularon 177 estudiantes, de los cuáles 63 pertenecen al Grado de Maestro/a en Educación Infantil y 114 al Grado de Maestro/a en Educación Primaria. De los cuales, un total de 170 estudiantes participaron en la evaluación del Prácticum I, a través de los cuestionarios, 60 de ellos del Grado de Maestro/a en Educación Infantil (35,3\%) y 110 del Grado de Maestro/a en Educación Primaria (64,7\%).

\section{METODOLOGíA Y/O INSTRUMENTOS UTILIZADOS}

La vía de evaluación del desarrollo del Prácticum I y II, es a través de cuestionarios cumplimentados por los estudiantes al reincorporarse a la actividad académica de la Facultad tras finalizar el periodo de prácticas en los centros educativos. Dichos cuestionarios se preparan y realizan por el Vicedecanato de Prácticas de la Facultad y aprobados por los miembros de la Comisión de Prácticas 
del Centro. El modelo de preguntas es el mismo para ambos grados y está divido en seis apartados con ítems correspondientes a: la Facultad de Educación, el acceso a la documentación del colegio, el periodo de rotación, las prácticas dirigidas, la valoración del centro y la valoración personal del desempeño en las prácticas. El cuestionario puede verse en el anexo correspondiente.

Se realiza el seguimiento de los miembros de los Programas de Maestro en Educación, cohortes 2016/2017 y 2017/2018, desde la asignación hasta la conclusión, mediante la tutoría personalizada, realizada por tutores de los Colegios Públicos y Concertados (CEIP), durante el proceso de formación en prácticas (PR1) y (PR2) en los CEIP de Castilla-La Mancha.

Tabla. Características demográficas de los participantes de los PE

\begin{tabular}{|c|c|c|}
\hline Variables demográficas & Media & \\
\hline Grado(s) & MEI & MEP \\
\hline $\begin{array}{l}\text { Estudios previos de } \\
\text { Educación }\end{array}$ & 0,2 & 02 \\
\hline Edad & 20,8 & 20,5 \\
\hline Dominio TIC & 3,5 & 3,7 \\
\hline $\mathrm{N}$ & (2G) 149 & 183 \\
\hline
\end{tabular}

Se emplearon cuestionarios, de papel y lápiz y en línea, para la valoración del proceso de práctica y las Destrezas de Enseñanza Efectiva (Santrock, J.W., 2002) con base en el cuestionario administrado al inicio del proceso de formación.

Se obtiene la autoevaluación de las destrezas de enseñanza organizadas en las tres dimensiones de Destreza Efectiva. Dichas destrezas están organizadas en tres dimensiones, en un cuestionario de 15 ítemes, tipo Likert (5), organizadas en: Conocimiento profesional y destrezas (8 ítems); II. Compromiso (3 ítems); y III. Crecimiento personal (4 ítems). Se autoevalúan también al final del programa.

\section{RESULTADOS ALCANZADOS}

Se aprecia la evolución en las Destrezas de Enseñanza Efectiva de los futuros profesionales de la Educación, el dominio de las competencias establecidas en el currículo de los Grados, el logro de los principios que componen el Estándar Profesional, seguidos en el ámbito internacional; así como satisfacción con el proceso de formación recibida, mediante la valoración de la experiencia, así como recomendaciones para la mejora.

\section{Formación Inicial de Profesionales de la Educación. Nuevo ingreso}

Se obtienen los resultados de los estudiantes de nuevo ingreso en el Grado en Maestro en Educación Infantil (MEI) y Primaria (MEP) en el Cuestionario de Autovaloración de Destrezas de Enseñanza Efectiva (DEE), de Santrock, J. W. (2002), al principio del curso escolar (pretest) y al final del PE. 
Gráfico 1. . Medias de autovaloración de Enseñanza Efectiva de estudiantes del Grado ME

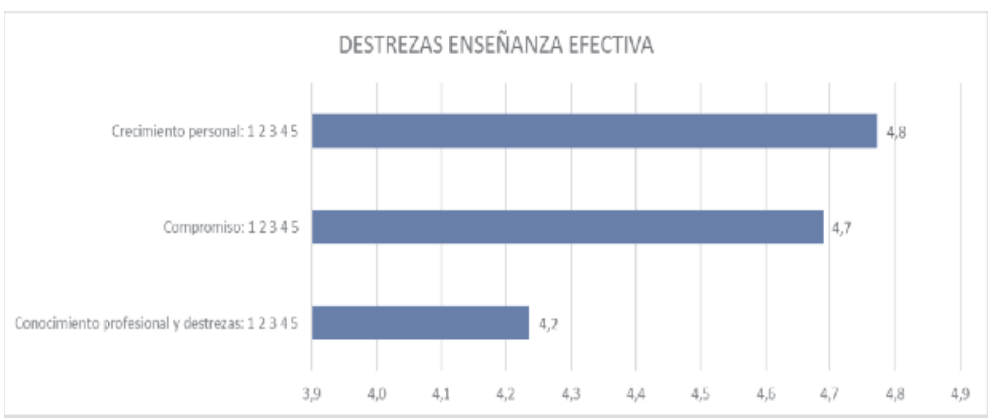

Se analizan las respuestas a los ítemes de las dimensiones: Conocimiento, Compromiso y Crecimiento Personal del cuestionario de Enseñanza Efectiva, se agruparon en rangos, bajo, medio y alto y se presentan los resultados en la tabla siguiente:

Tabla 1. Tabla de Dimensiones de Enseñanza Efectiva organizadas en las 3 Dimensiones: Conocimiento, Compromiso y Crecimiento Personal (niveles: bajo, medio y alto)

\begin{tabular}{|c|c|}
\hline Conocimiento Bajo & 1.0 \\
\hline Conocimiento Medio & 16.3 \\
\hline Conocimiento Alto & $\underline{81.7}$ \\
\hline Sin compromiso & 1.0 \\
\hline Comprometido & 10.6 \\
\hline Muy comprometido & $\underline{87.5}$ \\
\hline $\begin{array}{l}\text { Crecimiento Personal } \\
\text { Medio }\end{array}$ & 8.7 \\
\hline Crecimiento Personal Alto & $\underline{90.4}$ \\
\hline
\end{tabular}

Como se puede observar los estudiantes destacan en las tres dimensiones, en los rangos medios y altos.

Se presentan algunos aspectos, tomados del Informe (2017-2018), de la valoración de los periodos de PRACTICUM I y II, en las tablas y gráficos siguientes. 
Tabla 2. Evaluación del Prácticum I

\begin{tabular}{|c|c|c|c|c|}
\hline $\begin{array}{l}0 \\
\text { Nada } \\
\text { satisfactoria }\end{array}$ & $\begin{array}{c}1 \\
\text { Bie } \\
\text { n }\end{array}$ & $\begin{array}{l}2 \\
\text { Satisfactor } \\
\text { ia }\end{array}$ & $\begin{array}{c}3 \\
\text { Muy } \\
\text { Satisfactor } \\
\text { ia }\end{array}$ & $\mathbf{N S} / \mathbf{N C}$ \\
\hline \multicolumn{5}{|c|}{$\begin{array}{l}\text { I-6.1. Las tareas realizadas durante el periodo de prácticas han sido de } \\
\text { provecho para mi formación académica }\end{array}$} \\
\hline \multicolumn{5}{|c|}{$\begin{array}{l}\text { I-6.2. Considero que las prácticas han resultado útiles para mi desarrollo } \\
\text { personal (capacidad de comunicación, maduración, trabajo en equipo, } \\
\text { autoconfianza, etc...) }\end{array}$} \\
\hline \multicolumn{5}{|c|}{$\begin{array}{l}\text { I-6.3. Considero que las prácticas en los centros educativos son un buen } \\
\text { método para introducir al estudiante en el mundo laboral }\end{array}$} \\
\hline \multicolumn{5}{|c|}{ I-6.4. Considero que la duración del Prácticum I es apropiada } \\
\hline
\end{tabular}

\section{NS/NC}

I-6.1. Las tareas realizadas durante el periodo de prácticas han sido de provecho para mi formación académica

1-6.2. Considero que las prácticas han resultado útiles para mi desarrollo personal (capacidad de comunicación, maduración, trabajo en equipo, autoconfianza, etc...)

I-6.3. Considero que las prácticas en los centros educativos son un buen método para introducir al estudiante en el mundo laboral

I-6.4. Considero que la duración del Prácticum I es apropiada

Con relación al ítem: Las tareas realizadas durante el periodo de prácticas han sido de provecho para mi formación académica; en general, todos tienen una valoración positiva y buena ante este ítem, el $79 \%$ de los estudiantes han respondido que muy satisfactoria, ninguno ha respondido de forma negativa.

Gráfico 2. Valoración de las prácticas para la formación académica.

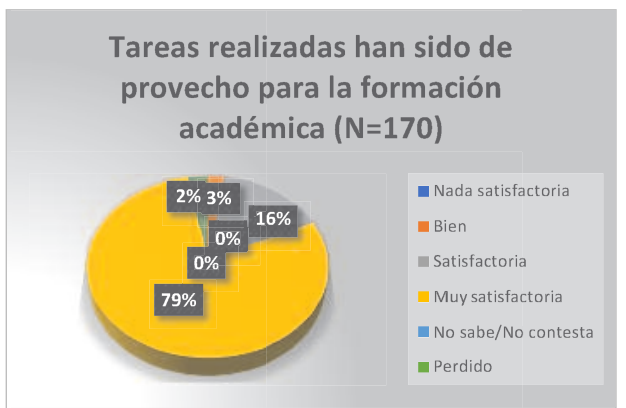


En el ítem: Considero que las prácticas han resultado útiles para mi desarrollo personal (capacidad de comunicación, maduración, trabajo en equipo, autoconfianza, etc...), la mayoría, el 89\% han respondido que muy satisfactoria, seguido del $8 \%$ que han mostrado que satisfactoria. El $3 \%$ se corresponde con los datos perdidos.

En el ítem: Considero que las prácticas en los centros educativos son un buen método para introducir al estudiante en el mundo laboral, las valoraciones son muy positivas. El $92 \%$ de los estudiantes han respondido de forma muy satisfactoria y el $5 \%$ con satisfactoria. El $3 \%$ se corresponde con los estudiantes que no han respondido.

Gráfico 4. Periodo de prácticas como método para inducir al mundo laboral.

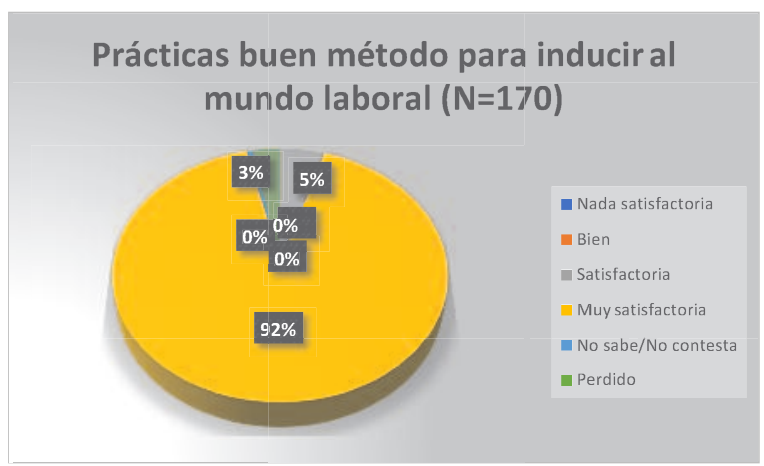

\section{VALORACION DEL PRACTICUM II}

Del conjunto de los 183 alumnos matriculados en los Grados de MEI y MEP han respondido un total de 170 (habiendo sido eliminadas las respuestas duplicadas), lo que se corresponde con un $85,8 \%$ del alumnado. Concretamente han respondido un total de 54 alumnos de GMEl y 103 alumnos de GMEP. En lo relativo a las menciones cursadas por el alumnado, se puede observar de los datos que las más solicitadas son: Pedagogía Terapéutica (PT) en GMEP (22\%), Educación Física en GMEP (18\%) y Necesidades Educativas Especiales (NEE) en GMEI (12\%). Un 1,2 \% afirman no haber cursado ninguna mención.

\section{SATISFACCION GENERAL CON EL PROGRAMA DE PRÁCTICAS}

Un total de 84 alumnos $(52,17 \%)$ han calificado las tutorías como muy satisfactorias mientras que un total de 69 alumnos (42,85\%) las han valorado entre satisfactoria y bastante satisfactoria.

Gráfico 5. Valoración de la organización de las tutorías

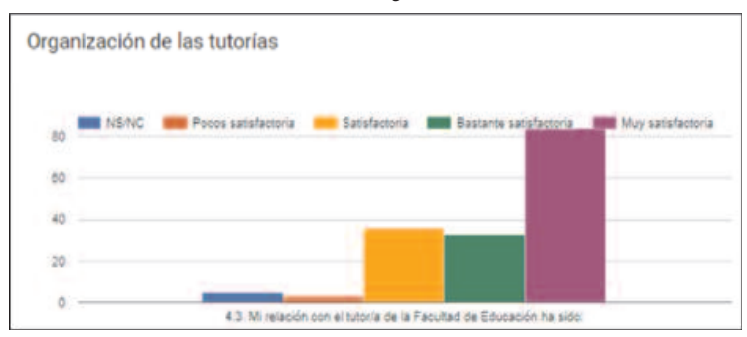




\section{SATISFACCION CON EL PROGRAMA DE PRÁCTICAS}

Gráfico 6. Nivel de Satisfacción con el Programa de Prácticas.

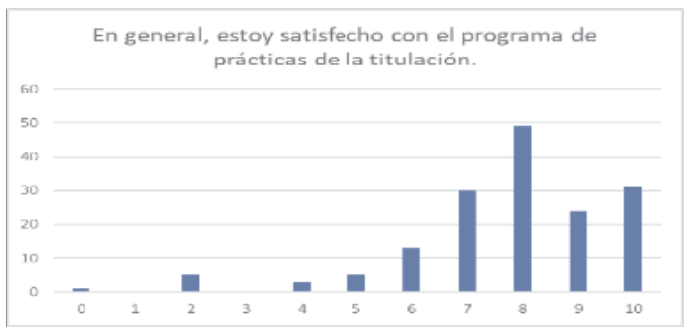

Con base en el gráfico anterior, se puede afirmar que en su gran mayoría el alumnado está satisfecho con el programa de prácticas, valorado por 134 alumnos $(83,23 \%)$ con una nota de entre 7 y 10.

En cuanto al centro de prácticas, un total de 67 alumnos $(41,61 \%)$ valora las tareas realizadas con un 10 , y 85 alumnos (52,8\%) valoran dichas actividades con una nota de entre 6 y 9 . Se puede afirmar, por lo tanto, que el alumnado ha estado bastante satisfecho con el periodo de prácticas y la labor de los centros y la facultad.

Gráfico 7. Satisfacción con las tareas realizadas en el colegio de prácticas.

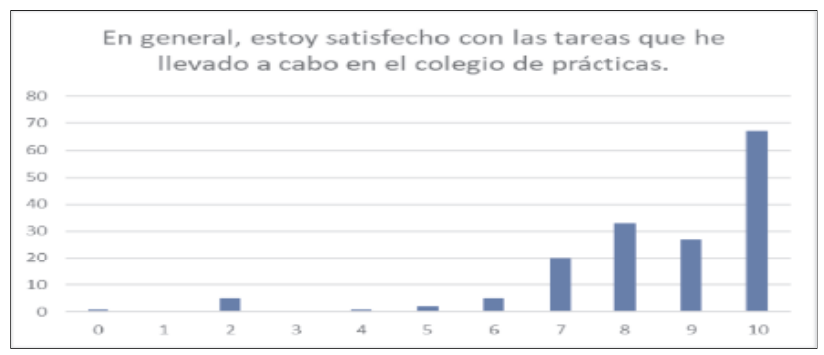

\section{RESULTADOS DE ENSEÑANZA EFECTIVA}

Se han obtenido las medias de los resultados de los estudiantes de nuevo ingreso en los ítemes del Cuestionario de Destrezas de Enseñanza Efectiva (DEE) del Programa Educativo, de los Grados MEI y MEP. 
Tabla 3 Inventario de Destrezas de Enseñanza Efectiva

Escala: 12345

Medias $1 \quad$ Medias 2

Conocimiento profesional y destrezas:

4,2

1. Tendré un excelente conocimiento y comprensión de la materia.

3,94 ,

2. Usaré estrategias instruccionales efectivas.

$4,0 \quad 5$

3. Tendré una excelente capacidad de planeación y para establecer objetivos.

4,2
9

4. Contaré con destrezas de manejo de grupo sobresalientes. 3

$3,8 \quad 4$

5. Seré capaz de ayudar a los alumnos a motivarse.

4,7

5

6. Tendré excelentes destrezas de comunicación.

4,

6. Tendré excelentes destrezas de comunicación.

$4,4 \quad 4$,

7. Trabajaré eficazmente con niños provenientes de ambientes culturales diferentes.

$4,6 \quad 5$

8. Contaré con excelentes destrezas en el manejo de $\quad 4,2$ tecnología.

$\underline{4,7}$

\begin{tabular}{lll} 
Compromiso: & $\underline{\mathbf{4 , 7}}$ & \\
\hline 9. Estaré extremadamente automotivado & 4,6 & 5 \\
10. Tendré una actitud estable & 4,7 & 4,7 \\
$\begin{array}{l}\text { 11. Contaré con destrezas adecuadas para interesarme por } \\
\text { los demás }\end{array}$ & 4,8 & 5 \\
\hline
\end{tabular}

\section{Crecimiento personal:}

4,8

12. Desarrollaré una identidad profesional positiva y bien integrada

13. Buscaré activamente el consejo de maestros competentes y experimentados

$\begin{array}{cc}4,8 & 5 \\ 4,7 & 4,7 \\ 4,9 & 4,9 \\ 4,7 & 4,7\end{array}$

14. Nunca dejaré de aprender

15. Construiré un sistema de apoyos y recursos sobresaliente.

\section{VALORACIONES COMPLEMENTARIAS DEL IMPACTO DE LAS PRÁCTICAS, DE TIPO CUALITATIVO}

"Desde mi punto de vista, debería de durar mucho más el programa de prácticas externas, ya que es en el colegio y el contexto escolar donde nos desarrollamos más, nos desenvolvemos mejor y adquirimos una experiencia muy enriquecedora que nos ayuda a avanzar y mejorar como docentes."

"Me gustaría comentar que el colegio y el personal han sido y son cruciales en mi aprendizaje como maestro. Siempre se han prestado a todo, con toda la confianza y la mejor de las sonrisas han contribuido a que me encuentre muy integrado y muy a gusto en el centro, y a que haya disfrutado muchísimo los periodos de prácticas. Una auténtica gozada, desde el director hasta la última de las maestras. 
"El año pasado también hice las prácticas con ellos (con la misma Maestra también) y, puesto que acabé tan contento y el periodo de prácticas era tan corto, seguí yendo el resto del año todas las horas libres que tenía. En el colegio siempre me reciben con los brazos abiertos y sin ningún problema. Este año, ahora que ya acabamos y el mes de marzo lo tengo completamente libre, seguiré yendo también al colegio como práctico".

"Solamente quería mostrar mi agradecimiento con el colegio, con su director y con mi maestratutora en particular y que quede patente que me han ofrecido el ambiente idóneo para mi formación".

"Lo único negativo que señalaría es la corta duración del periodo de prácticas. Pienso que es donde el alumnado realmente se vale como futuros docentes y donde se pone en práctica todo lo aprendido a lo largo de estos 4 años".

"Tanto mi tutor de la facultad como mi tutora del colegio han sido muy atentos conmigo. En el Colegio he podido estar con mi maestra de clase y con los diferentes especialistas como es el profesorado de inglés, educación física y la logopeda. Me han dejado dar las clases que yo quisiera (dentro y fuera de mi unidad) y además he participado casi desde el principio en las explicaciones de las actividades del aula casi todos los días. Además, cuentan conmigo para realizar diferentes actividades como las fiestas del colegio y alguna excursión que hay fuera de fecha del prácticum para que pueda seguir formándome y conocer más cosas del colegio."

\section{DISCUSIÓN}

Sin duda, es muy importante el análisis continuo de la información, seguimiento y la valoración de la tutoría de los CEIP y de los tutores de la Universidad como fuente de retroalimentación de las acciones formativas de los PE de Educación;

En adecuado el empleo de un modelo de estudio, tipo pretest-postest, ya que es necesaria la valoración de las Destrezas de Enseñanza Efectiva, al ingreso y al final de los estudios de Grado y contrastar con un Modelo de tipos de Conocimiento que debe dominar el Profesor, como propone Shulman, L. S., (1986) y un Estándar de Formación de Profesores, como el empleado en otros contextos, como el INTASC, y relacionar también con el bienestar, de tipo psicológico, de los futuros profesionales de la educación en los ámbitos de la educación infantil y la educación primaria.

También es recomendable destacar en la formación de los Profesionales de la Educación Infantil y Primaria, el seguimiento de los Principios expresados en el documento de la American Psychological Association (APA), Coalition for Psychology in Schools and Education. (2015). 20 principios para la Enseñanza y el Aprendizaje, desde la Educación Infantil al Grado 12 (Top 20 principles from psychology for preK- 12 teaching and learning); disponible en http://www.apa. org/ed/schools/cpse/top- twenty-principles.pdf

\section{CONCLUSIONES}

Del estudio se derivan conclusiones parciales sobre el desarrollo de las Destrezas de Enseñanza Efectiva en los futuros profesionales de la Educación, por lo que el propósito es continuar con estudios con miras a obtener el panorama má completo, a partir de seguimiento del desarrollo de las competencias en la práctica real de los docentes, noveles y en ejercicio, como sugieren autores, como Marcelo, C., siguiendo las ideas de Shon, Shulman, Zeichner entre otros investigadores con estudios reconocidos sobre la temática de la Formación de Profesores.

\section{Prospectiva.}

En el ámbito de la Unión Europea, los Estados miembros y la Comisión Europea (Consejo de la Unión Europea, 2014), invitan a: "hacer el mejor uso de las estructuras ET 2020 en el marco del método abierto de coordinación para fortalecer la formulación de políticas basadas en la evidencia 
y desarrollar y difundir la prácticas con éxito en: i) Programas de formación docente efectiva, con énfasis en la formación inicial del profesorado y formas en que deben abordarse los enfoques de colaboración en las acciones formativas; ii) El desarrollo profesional de los formadores de FP en empresas, dado su papel crucial en proporcionar aprendizajes de alta calidad y otras formas de aprendizaje en el trabajo; iii) Reforzar la relevancia de los currículos de formación docente y las competencias profesionales de profesores y formadores, en su caso a través de una cooperación más estrecha con los empleadores, incluidas las empresas.

Así como, aprovechar los resultados de estudios y encuestas relevantes, lo que invita a consolidar en la FE de la UCLM líneas de trabajo e investigación siguiendo dichas políticas.

En particular, en el curso 2019-2020, se buscará relacionar dicho proceso de formación con el Bienestar Psicológico -Well-Being, de los profesionales de la Educación a punto de egresar, como una línea de investigación del grupo EMOCOG, compuesta por miembros del Departamento de Psicología de la UCLM, empleando la adaptación del instrumento del Modelo de Ryff, C. realizado por Díaz, D., et al. (2006), modelo seguido en diferentes investigaciones en proceso.

\section{REFERENCIAS BIBLIOGRÁFICAS}

American Psychological Association, Coalition for Psychology in Schools and Education. (2015). Top 20 principles from psychology for preK-12 teaching and learning. Disponible en http://www.apa. org/ed/schools/cpse/top-twenty- principles.pd

Consejo Unión Europea, (mayo 2014). Conclusiones sobre formación docente eficaz. En: https://intef.es/Blog/conclusiones-sobre-formacion-docente-eficaz-consejo- union-europeamayo-2014/

Comisión Europea (2019). Informe de julio 2013 del Grupo de Trabajo Temático sobre Desarrollo Profesional Docente ("Supporting teacher competence development for better learning outcomes“). En https://ec.europa.eu/education/ Consultado: 01-02-2019

Darling-Hammond, L. (2012). Desarrollo de un enfoque sistémico para evaluar la docencia y fomentar una enseñanza eficaz. Pensamiento Educativo, 49(2), 1-20. https://doi.org/10.7764/PEL.49.2.2012.1

FE-UCLM (2017). Informe de PRÁCTICUM I (2017-2018). UCLM FE CR:

Vicedecanato de Prácticas: Informe interno.

FE-UCLM (2018). Informe de Evaluación del periodo de PRÁCTICUM II 2017/2018.

Grados: MEI y MEP. UCLM-FE-CR: Informe interno.

Facultad de Educación. C. R. -UCLM (2018). Orientaciones Generales para las Prácticas de Enseñanza. En: http://www.uclm.es/cr/educacion/index.asp Consultado 30/04/2018

Garrido, C. A., \& Torrecilla, F. J. (2016). Estudio sobre la Enseñanza Eficaz y su impacto sobre el desarrollo de los estudiantes. Recuperado el 10 de 5 de 2019, de https://dialnet.unirioja.es/servlet/articulo?codigo $=6405045$

INTASC (2019). InTASC Model Core Teaching Standards and Learning Progressions for Teachers 1.0 Council of Chief State School Officers (CCSSO). En: https://ccsso.org/resource-library/intascmodel-core-teaching-standards-and- learning-progressions-teachers-10 Consultado $14 / 05 / 2019$

PRAXIS II (2019). En Woolfolk (2014) Psicología Educativa 9ª Apéndice. Pp 581-596.

Ed. Pearson Educación. Educational Testing Service (ETS). Disponible en: https://www.ets.org/praxis

Laudadío, M. J., \& Da Dalt, E. (2014). Estudio de los estilos de enseñanza y estilos de aprendizaje en la universidad. Educación y Educadores, 17(3), 483-498. https://doi.org/10.5294/ edu.2014.17.3.5 
Perfil diferencial de la competencia docente psicoinstruccional entre el cuerpo de maestros y el de profesores de enseñanza secundaria del sistema educativo español actual. (2012). International Journal of Developmental and Educational Psychology: INFAD. Revista de Psicología, (1), 427. Retrieved

from http://search.ebscohost.com/login.aspx?direct=true \&db=edsdnp\&AN=edsdnp.52 29522ART\&lang=es\&site=eds-live

Martínez-Garrido, C. (2015). Investigación sobre enseñanza eficaz. Un estudio multinivel para Iberoamérica. Director: F.J. Murillo. Facultad de Formación de Profesorado y Educación de la Universidad Autónoma de Madrid. Madrid.

Recuperado el 10 de 5 de 2019, de http://revistaselectronicas.ujaen.es /index.php/ade/article/view/3491/3028

Martínez-Garrido, C. y Murillo, F.J. (2016). Investigación Iberoamericana sobre Enseñanza Eficaz. Revista Mexicana de Investigación Educativa, 21(69), 471-

499. (Scopus)

Murillo, F. J., Martínez Garrido, C. y Hernández-Castilla, R. (2011). Decálogo para una enseñanza eficaz. REICE. Revista Iberoamericana sobre Calidad, Eficacia y Cambio en Educación, 9(1), 6-27.

Pérez Abril, M.; Roa Casas, C.; Patricia Vargas, Á.; \& Isaza, Lida A. (2014). What Makes an Outstanding Teacher? Features of Practice during the First Years of School. Revista Colombiana de Educación, (67), 171-200. Retrieved May 10, 2019, from http://www.scielo.org.co/scielo.php?script=sci_arttext\&pid=S012039162014000200009\&lng=en\&tlng=en.

Posner, Ch. M. (2004). Enseñanza efectiva: una revisión de la bibliografía más reciente en los países europeos y anglosajones. Red Revista Mexicana de Investigación Educativa. vol. 9, núm. 21, abril-junio, 2004, pp. 277-318. Consejo Mexicano de Investigación Educativa, A.C.

Román, M. (2010). Investigación latinoamericana sobre enseñanza eficaz. Recuperado el 10 de 5 de 2019, de http://idep.edu.co/revistas/index.php/educacion-y- ciudad/article/download/121/110

Ventura, A. C. (2016). ¿Enseño como aprendí?: el rol del estilo de aprendizaje en la enseñanza del profesorado universitario. Aula Abierta, 44(2), 91-98. https://doi.org/10.1016/j.aula.2016.05.001

Santrock, J. W. (2002). Psicología de la Educación. México: McGraw-Hill Interamericana, SA de CV Struthers, C. W., \& Perry, R. P. (1993). Effective Teaching in the College Classroom: Current Perspectives and Future Directions. Revista Española de Pedagogía,

51(194), 5-26. Retrieved from http://search.ebscohost.com/login.aspx? direct=true \&db=lsdpd\&AN=29351\&lan $\mathrm{g}=\mathrm{es} \&$ site $=$ eds-live

Schön, D. (1992). The Reflective Practitioner. London: Routledge, https://doi.org/10.4324/9781315237473

Shulman, L. S. (1986). Those Who Understand: Knowledge Growth in Teaching.

Educational Researcher, 15(2), 4-14.En https://doi.org/10.3102/0013189X015002004Schon

Stufflebeam, D. L.; Coryn C. L. (2014). Evaluation Theory, Models, and Applications.

John Wiley \& Sons.

Torrecilla, F. J. (2003). Una panorámica de la investigación iberoamericana sobre eficacia escolar. Recuperado el 10 de 5 de 2019, de http://inacap.com/tportal/portales/tp4964b0e1bk102/uploadimg/file/teoriapracte ns/practicasens/6_efectividadeducativa.pdf

Torrecilla, F. J., Garrido, C. A., \& Castilla, R. H. (2011). Decálogo para una enseñanza eficaz. Recuperado el 10 de 5 de 2019, de https://repositorio.uam.es/handle/10486/661375

Woolfolk, A. (2014). Psicología de la Educación. España: Pearson 28/02/201 